\title{
Enabling hospital staff to care for people with dementia
}

\author{
In the final article of this series, Jennifer Bray and colleagues \\ present two case studies that show how awareness training and \\ standardised pain assessment have led to improved understanding
}

Correspondence

j.bray@worc.ac.uk

Jennifer Bray is research assistant

Simon Evans is principal research fellow and head of research

Mary Bruce is senior lecturer

Christine Carter is senior lecturer

Dawn Brooker is director of the Association for Dementia Studies

Sarah Milosevic is former research assistant

All at University of Worcester

Rachel Thompson is professional and practice development lead for Admiral Nursing, Dementia UK and member of the Nursing Older People editorial advisory board

Catherine Woods is nurse specialist in pain management, Nottingham University Hospitals NHS Trust

Date of submission

March 92015

Date of acceptance June 252015

Peer review

This article has been subject to double-blind peer review and has been checked using antiplagiarism software

Author guidelines journals.rcni.com/r/ nop-author-guidelines

\begin{abstract}
This is the fourth and final article in a short series that presents case study examples of the positive work achieved by trusts who participated in the Royal College of Nursing's development programme to improve dementia care in acute hospitals.

Dementia training in hospitals is often inadequate and staff do not always have sufficient knowledge of dementia to provide appropriate care. It can also be difficult for them to identify when patients with dementia are in pain, especially when their communication skills deteriorate. The case studies presented illustrate how two NHS trusts have worked to ensure that their staff are fully equipped to care for people with dementia in hospital.
\end{abstract}

IN LINE with the SPACE principle that there should be 'skilled staff who are informed and have enough time to care (Thompson and Heath 2013) (Box 1), Basildon and Thurrock University Hospitals NHS Foundation Trust in Essex identified increasing staff knowledge and awareness of dementia as a priority in their involvement with the RCN dementia development programme. Their aim was to ensure all staff were made aware of the needs of patients with dementia, and to achieve this they updated and improved their dementia training programme.

It is widely recognised that training in dementia care is often inadequate in hospitals. A national survey found that while $54 \%$ of nursing staff had not received any pre-registration training and only $12 \%$ thought they had received enough training, $83 \%$ felt that learning and development opportunities would help them to provide care for people with dementia (Alzheimer's Society 2009). It is also recommended that 'any training
Basildon and Thurrock University Hospitals NHS Foundation Trust in Essex made dementia training a priority by including dementia awareness in staff induction across a range of roles and providing additional training activities tailored to meet staff needs. Nottingham University Hospitals NHS Trust focused on pain assessment, aiming to standardise its approach for patients with dementia. The pain assessment in advanced dementia tool was chosen and piloted, and is being implemented across the trust after a positive response.

\section{Keywords}

acute hospitals, dementia, development programme, pain assessment tools, staff training

strategy needs to include other front line staff, ambulance crews working in patient transport, healthcare assistants, porters and catering staff. All these groups should have some knowledge and understanding of dementia' (NHS Confederation 2010).

Consequently, the training planned by Basildon Hospital was aimed at staff across all roles including receptionists, porters, care support workers and staff nurses, with different levels of information and detail being provided as appropriate to each role. A first step was to embed a brief session of dementia awareness training in the staff induction programme, although it was recognised that this would take time to filter through to all staff because it focused on new staff.

Dementia has also become part of the mandatory induction process for agency staff before their first shift, however, because cleaning staff are employed by a private contractor they have not completed this training. Medical staff are not involved in the 
training, but foundation year 1 and 2 doctors have previously been provided with a 45-minute dementia awareness training session and will be required to complete a basic awareness e-learning package before they begin working.

\section{Dementia awareness training}

As of March 2014, 219 staff across four elderly care wards had attended in-depth training with most being care support workers or staff nurses (Figure 1).

The training covered topics such as:

Recognising the symptoms of dementia.

- Recognising the signs of depression.

- Communicating with patients with dementia and their carers.

- Psychosocial interventions.

Person-centred care.

Behaviour that challenges.

Pain assessment.

Use of relevant tools including cognitive assessment tools.

This in-depth training is based on a package from the University of Stirling which includes DVDs that follow patients with dementia through their journey and present carer stories. However, the training session only lasts three hours so there is not enough time to show all of the films.

The team involved in the RCN dementia development programme witnessed a number of changes across the hospital. Their observations, combined with the findings from evaluation questionnaires completed by staff before and after the training, indicate that the training had an effect on staff behaviour and opinions. The evaluation questionnaires found that most staff would be 'extremely likely' or 'very likely' to put into practice what they had learned, and staff had an increased understanding and awareness of dementia.

By emphasising the need to appreciate patients as individuals rather than focusing solely on treatment options, the training at Basildon Hospital has supported a shift away from using a medical model to a more psychosocial approach to care. This has made a significant difference to how staff respond to the behaviour of patients with dementia, and it is now common to see staff joining patients on a walk around a ward area instead of telling them to sit down as they might have done previously.

The team has seen that staff have a better understanding of why they may need to work more closely with patients with dementia and how greater interaction can help address different behaviours. For example, carers are encouraged to bring in items from home which can be used by staff to

\section{Box 1 SPACE principles}

There are five principles for the care of people with dementia in hospital settings, developed in collaboration with people with dementia and carers. Each principle has a set of sub-statements that can be used to assess progress and identify areas for further development. The principles are:

Skilled staff who are informed and have enough time to care

Partnership working with carers

Assessment and early identification of dementia

Care plans that are person-centred and individualised

Environments that are dementia friendly

(Thompson and Heath 2013)

engage with patients and there is a greater focus on providing meaningful activities.

It has also been noticed that staff are sharing information more freely outside of the training sessions, such as mental health liaison workers passing on relevant information about patients to other staff members. The increased awareness of dementia among staff has also helped with cross-departmental working, and a group of clinicians meets monthly to help address potential issues relating to patients with dementia.

Despite the improvements made so far, staff at Basildon Hospital are not slowing down; they are already considering plans to develop their training programme even further, with the hope of involving carers in their training delivery. They plan to do this by encouraging carers to share their own experiences of the hospital care provided to their relatives and to state what effect it had on them. When personal stories have been used previously in the hospital's training, they have proved to be powerful for staff.

Staff on the elderly care wards have identified a need for more in-depth training on behaviour that challenges, and this is being developed.

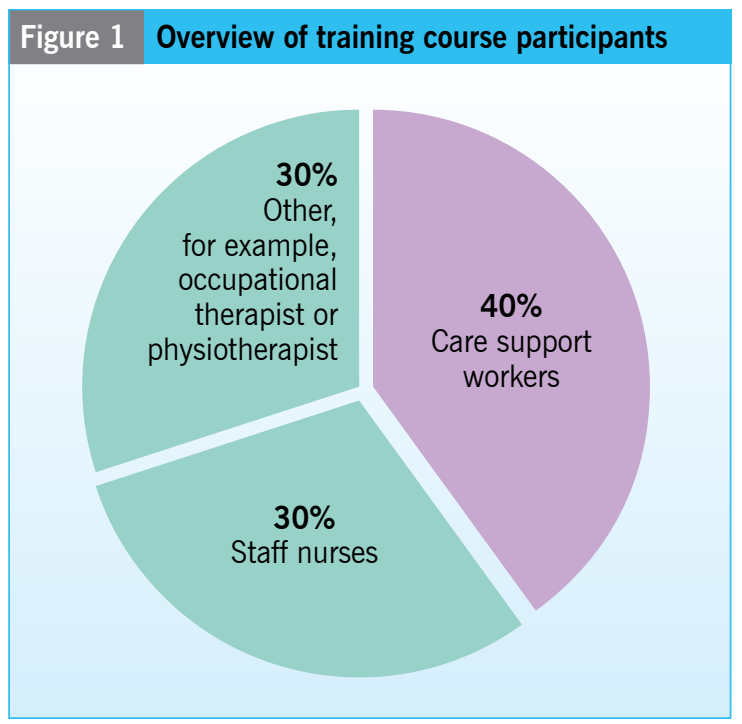


To enhance future evaluations of the training programme the 20-item dementia knowledge questionnaire DK-20 (Shanahan et al 2013) and the sense of competence in dementia care staff scale (Schepers et al 2012) will be used before and after each training course to assess whether knowledge of dementia has improved.

The training programme developed and implemented by Basildon Hospital had an ambitious aim. By recognising that different staff require different levels of training and adapting their provision accordingly, they have been able to target different groups using appropriate methods which has helped to increase staff understanding and awareness of dementia. This has been an important factor in helping to change hospital culture, which in turn is having a noticeable effect on the way staff interact with patients who have dementia.

\section{Piloting a pain assessment tool}

As the RCN recognises, 'pain is one of the most common but under-recognised and under-treated symptoms in dementia' (Thompson and Heath 2013). When dementia reduces a person's language skills it becomes more difficult to know what that person is experiencing, making observations of body language and behaviour more important (Cunningham et al 2010).

Consequently, one of the RCN SPACE principles states that person-centred care plans should involve the use of 'dementia appropriate tools, for example, assessment of nutrition, pain, function, risk etc' (Thompson and Heath 2013).

This more detailed observation of patients with dementia depends on sufficient awareness and understanding among nursing staff. A survey found that $71 \%$ of nursing staff thought that more training in being able to recognise pain in patients with dementia was 'needed or vital' (Alzheimer's Society 2009).

When Nottingham University Hospitals (NUH) NHS Trust took part in the RCN dementia development programme, it was identified that both of its hospitals needed to improve their practice for pain assessment in patients with dementia.

An internal survey of existing practice at Nottingham City Hospital and Queen's Medical Centre found that there was no standard method for assessing pain when caring for patients with dementia, including those with communication difficulties. Some wards used the Abbey Pain Scale (Abbey et al 2004), while other wards did not use a dementia-specific tool. The trust sees pain assessment as a priority for all patients and recognises that patients who cannot communicate pain verbally are potentially at greater risk of their pain issues being unrecognised and unaddressed.

To address this, the trust's nurse specialist in pain management developed an action plan in September 2013 for standardising pain assessment for patients with dementia. The first part of the plan involved conducting a short survey of 85 adult nursing staff to explore what they thought would help them improve their ability to assess pain in patients with dementia, in particular those who have difficulty communicating.

The survey revealed that:

- $91 \%$ of staff thought that having a pain assessment tool would help them.

89\% said a tool would improve their practice.

- $83 \%$ said that having a tool would make them feel more confident in raising pain as an issue with medical colleagues.

-50\% said they were unsatisfied with current practices.

In addition, staff thought a standardised approach to pain assessment and staff education would help to improve patient care.

After the survey, a literature review was initiated by the nurse specialist in pain management at Nottingham City Hospital to identify appropriate pain assessment tools. The literature search was undertaken by the NUH library service through the Cumulative Index to Nursing and Allied Health Literature database using the search words 'pain, assessment and dementia'. In addition, key documents were reviewed for recommendations about practice. The following shortlist of tools was produced:

- Pain Assessment in Advanced Dementia - PAINAD (Warden et al 2003).

- Pain Assessment Checklist for Seniors with Limited Ability to Communicate - PACSLAC (Fuchs-Lacelle and Hadjistavropoulos 2004). DOLOPLUS 2 (Lefebvre-Chapiro 2001).

Checklist of Non-verbal Pain Indicators - CNPI (Feldt 2000).

Abbey Pain Scale (Abbey et al 2004).

Mobilization-Observation-Behavior-IntensityDementia-2 - MOBID-2 (Husebo et al 2007). A multidisciplinary working group was formed of approximately 30 staff including nurses, healthcare assistants (HCAs), occupational therapists, discharge facilitators and physiotherapists. Their role was to decide which tool would be most appropriate for use in the acute hospital environment.

Each tool was evaluated by the group using a simple form designed by the nurse specialist in pain management to capture feedback on:

Ease of use.

Time taken to conduct an assessment using the tool. Practicality of the tool for the clinical area. 
Reliability.

Fitness for purpose.

Positives and negatives of the tool.

The responses were collated by the nurse specialist in pain management with the overall consensus being that the PAINAD tool was most suitable for piloting in the trust over a two-month period. To ensure that the tool was piloted on a variety of wards, dementia champions in the trust were approached and engaged in the process. Consequently, the PAINAD tool was trialled on surgical and medical wards, a health care of older people ward and in the short stay and admission units, mainly by nurses and HCAs.

To support the pilot, an evaluation survey was used to obtain feedback from staff about their use of the PAINAD tool. This survey was simple and covered areas such as ease of use/completion, time required to complete, actions taken, if anything was unnecessarily included in or missing from the tool and an overall rating of the tool.

In addition to the formal pain assessment tool, it was decided that carer knowledge should be capitalised on and captured in a more consistent fashion. Therefore, new questions have been added to the hospital's existing 'About Me' document, which carers are encouraged to complete on behalf of their relative with dementia. These additional questions ask carers to outline any signs that can be used to identify when their relative may be in pain, and what staff can do to try to ease that pain.

The findings from the two-month pilot phase were presented to NUH's Nursing and Midwifery Research Strategy Group in June 2014. It was reported that staff liked the PAINAD tool and found it quick and easy to use, and it was given a score of more than seven out of ten for usefulness. The work done so far around pain assessment is already seen as a positive development, and the PAINAD tool is in the process of being launched trust-wide. Nottingham City Hospital has been sharing its information with another trust taking part in the RCN dementia development programme, and other trusts have also expressed interest.

Although some work around pain assessment tools had been started in the trust before their inclusion in the RCN programme, it was limited to the progress that one member of staff could achieve by fitting it around their clinical role. Being part of the RCN dementia development programme provided more time, focus and structure on this subject, giving it a real impetus and helping it to be taken forward.

The literature review indicated that using a pain scale did not mean that the results were acted on and educational programmes to support their use were necessary to make sure that staff understood the reasons and importance behind them. With this in mind, the PAINAD tool will be launched trust-wide with a related education programme. Indeed, some teaching and promotion of the tool has already taken place.

The PAINAD tool will also be added to the trust's Pain Benchmark, with a view to auditing the tool after six months to ensure it is still fit for purpose.

Consistent use of an appropriate pain assessment tool by trained and knowledgeable nursing staff is vital to improving pain management for patients with dementia, especially as their language skills deteriorate. NUH NHS Trust has taken an important step towards achieving this and hopes to sustain and expand the use of the PAINAD tool in the future.

While the RCN development programme enabled the trust to focus on addressing pain assessment for patients with dementia, other important factors in the success of this project included having clear objectives, negotiating time to undertake the project and identifying early on who the key people were in terms of assisting with and influencing the work. The main challenge faced by the trust was securing staff time to carry out the work and to release staff from clinical areas to attend training. These elements should therefore be borne in mind by any other trusts wishing to carry out similar work around pain assessment for patients with dementia.

\section{Online archive}

For related information, visit our online archive and search using the keywords

Conflict of interest None declared

\section{References}

Abbey J, Piller N, De Bellis A et al (2004) The Abbey pain scale: a 1-minute numerical indicator for people with end-stage dementia. International Journal of Palliative Nursing. 10, 1, 6-13.

Alzheimer's Society (2009) Counting the Cost: Caring for People with Dementia on Hospital Wards. Alzheimer's Society, London.

Brooker D, Milosevic S, Evans S et al (2014) RCN Development Programme: Transforming Dementia Care in Hospitals - Evaluation Summary Report. University of Worcester, Worcester.

Cunningham C, McClean W, Kelly F (2010) The assessment and management of pain in people with dementia in care homes. Nursing Older People. 22, 7, 29-35.
Feldt K (2000) The checklist of nonverbal pain indicators. Pain Management Nursing. 1, 1, 13-21.

Fuchs-Lacelle S, Hadjistavropoulos T (2004) Development and preliminary validation of the pain assessment checklist for seniors with limited ability to communicate (PACSLAC). Pain Management Nursing. 5, 1, 37-49.

Husebo B, Strand L, Moe-Nilssen R et al (2007) Mobilization-Observation-Behavior-IntensityDementia Pain Scale (MOBID): development and validation of a nurse-administered pain assessment tool for use in dementia. Journal of Pain and Symptom Management. 34, 1, 67-80.

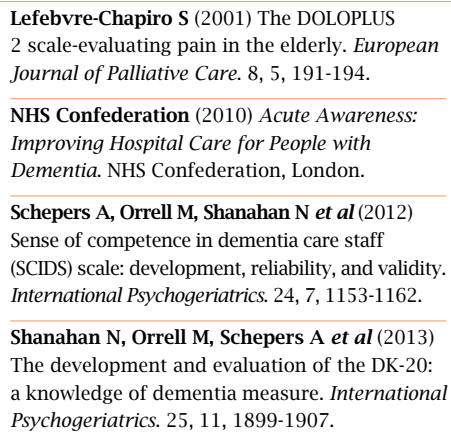

Lefebvre-Chapiro S (2001) The DOLOPLUS 2 scale-evaluating pain in the elderly. European Journal of Palliative Care. 8, 5, 191-194. NHS Confederation (2010) Acute Awareness: Improving Hospital Care for People with Dementia. NHS Confederation, London. Schepers A, Orrell M, Shanahan N et al (2012) Sense of competence in dementia care staff (SCIDS) scale: development, reliability, and validity. International Psychogeriatrics. 24, 7, 1153-1162.

Shanahan N, Orrell M, Schepers A et al (2013) The development and evaluation of the DK-20: a knowledge of dementia measure. International Psychogeriatrics. 25, 11, 1899-1907.

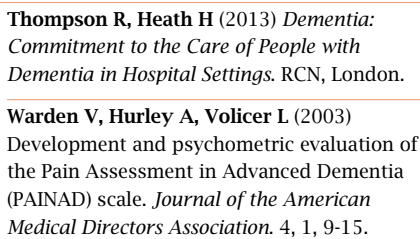
Dementia in Hospital Settings. RCN, London. Warden V, Hurley A, Volicer L (2003) Development and psychometric evaluation of the Pain Assessment in Advanced Dementia (PAINAD) scale. Journal of the American Medical Directors Association. 4, 1, 9-15. 
